\title{
Correction of Quality of Control Systems of Remotely Controlled Aerial Vehicles
}

\section{A.K. Ablesimov}

Aviation Computer-

Integrated Complexes

Department,

National Aviation University

Kyiv, Ukraine

alexander.ablesimov@gmail.com

\author{
M.A. Pylypenko \\ Aviation Computer- \\ Integrated Complexes \\ Department, \\ National Aviation University \\ Kyiv, Ukraine \\ pilipenko_63@ukr.net
}

\author{
A.M. Ryabokonev \\ Aviation Computer- \\ Integrated Complexes \\ Department, \\ National Aviation University \\ Kyiv, Ukraine \\ ryabokonev_a@ukr.net
}

\author{
K.A. Adamchuk \\ Aviation Computer- \\ Integrated Complexes \\ Department, \\ National Aviation University \\ Kyiv, Ukraine \\ adamchuk_katerina@ukr.net
}

Abstract-Analyzed the properties of corrective devices for remotely controlled aerial vehicles and are designed algorithms for calculating their frequency characteristics.

Keywords-correction device; inverse transfer function; block diagram;the desired frequency characteristics; logarithmic phase and frequen cy characteristics

\section{INTRODUCTION}

Remotely controlled aerial vehicles (RCAV) are increasingly used in human activities.

RCAV control systems may consist from different by physical nature and structure of elements. However, the regulation processes occurring in these systems are described by similar linear differential equations. As a result their structural schemes are identical, that allows to obtain a typical (Fig. 1) block diagram of a control system RCAV.

The following designations are accepted in structural scheme: $W_{0}(s)=\frac{k_{0}}{\left(T_{0} s+1\right) s}$ is the transfer function of a control object; $W_{\mathrm{op}}(s)=\frac{e^{-\tau s}}{\left(T_{\mathrm{op}} s+1\right)^{2}}$ is the transfer function of the operator; $k_{\text {ads }}$ is a static transfer coefficients of the angular deflection sensor; $k_{\mathrm{vds}}$ is a static transfer coefficients of the velocity deviation sensor; $k_{\text {reg }}$ a static transfer coefficients of the regulator.

Control and stabilization moment is formed by the channels of said sensors

$$
\bar{M}_{\mathrm{s}}=\bar{M}_{G}+\bar{M}_{D}
$$

General view of the equations of motion of system

$$
\mathrm{Y}(s)=W_{x}(s) R(s)+W_{y}(s) M_{y \Sigma}(s)
$$

where $W_{x}(s)$ and $W_{y}(s)$ are the transfer functions by the control signal and by the external disturbances

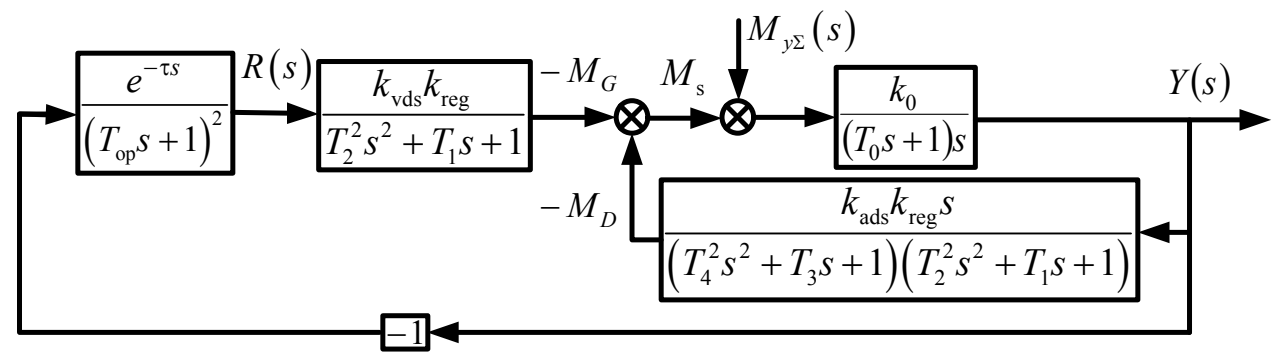

Fig. 1. Block diagram of RCAV control system.

The presence of the block diagram enables to perform analysis and synthesis of control systems for remotely controlled aerial vehicles.
The illustrated structural scheme does not always allow to obtain the required quality of the control processes at the selected design parameters of elements. Besides feedback by the 
absolute angular velocity of the control object into the structure of the regulator of the control system of RCAV are usually introduced an additional parallel and serial corrective devices to ensure a given quality.

\section{PROBlem Statement}

The use of a particular type of corrective device $(K U)$ is defined by the ability of its technical realization.

During the synthesis the developer have to choose the type $\mathrm{KU}$, proceeding from their properties, that are determined by the ways of their inclusions into the control system, perform calculations of the frequency characteristics of corrective devices, determine their schematics and design solutions, calculate the optimum parameters based on the requirements for quality to control systems, which are defined by specifications.

To ensure timely and quality execution of such work requires specific skills and experience.

The material in this article can be seen as some of the recommendations that may be useful for developer in the synthesis of the corrective devices of RCAV control systems.

\section{SOLUTION OF THE PROBleM}

Parallel corrective devices are implemented as rigid and flexible feedbacks covering an element, a chain of elements or the entire system. Hard negative feedbacks are used relatively rarely for the correction of quality. This is because that the introduction of negative rigid feedback, providing speed increasing and expansion of the linearity zone, at the same time reduces the transfer coefficient of the covered element or the chain of elements. As a result - increasing of the control errors.

The most widely for the purposes of quality correction are applied the flexible feedbacks - feedbacks from the derived output value of the element (a chain of elements) or system. Since the flexible feedbacks are acting only during the transients, their introduction allows to correct the quality of control without changing the static properties of the system.

In general, the inverse transfer function of the open-loop system, adjusted by the ideal differentiating feedbacks, has the form:

$$
E_{\mathrm{ol}}^{(\mathrm{c})}(s)=E_{\mathrm{ol}}(s)+k_{1} s+k_{2} s^{2}+k_{3} s^{3}+\ldots
$$

where $E_{\mathrm{ol}}(s)$ is the inverse transfer function of the system, that is not corrected; $k_{1}, k_{2}, k_{3}, \ldots$ are coefficients of flexible feedback of the corresponding order by dimension second.

Inverse amplitude and phase frequency characteristic (APFC) of the corrected system according to equation (1) will be:

$$
E_{\mathrm{ol}}^{(\mathrm{c})}(j \omega)=U_{E}^{(\mathrm{c})}(\omega)+j V_{E}^{(\mathrm{c})}(\omega),
$$

where

$$
\begin{aligned}
& U_{E}^{(\mathrm{c})}(\omega)=U_{E}(\omega)-k_{2} \omega^{2}+k_{4} \omega^{4}-\ldots ; \\
& V_{E}^{(\mathrm{c})}(\omega)=V_{E}(\omega)+k_{10} \omega-k_{3} \omega^{3}+\ldots
\end{aligned}
$$

Therefore, the coordinates of each point of the original characteristic $E_{\mathrm{ol}}(j \omega)$ with the introduction of flexible feedbacks acquire an increments

$$
\begin{aligned}
& \Delta U_{E}(\omega)=-k_{2} \omega^{2}+k_{4} \omega^{4}-\ldots, \\
& \Delta V_{E}(\omega)=k_{1} \omega-k_{3} \omega^{3}+\ldots,
\end{aligned}
$$

and the whole characteristic is shifted on the plane $U_{E}(\omega) \rightarrow j V_{E}(\omega)$.

Figure 2 shows the feedback APFC deformation of the open loop system when introduced the four first derivatives of control value.

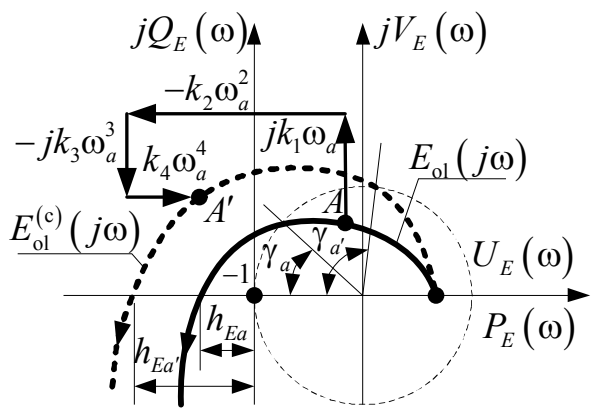

Fig. 2. The offset of the point of inverse APFC of the open-loop system $E_{\mathrm{ol}}(j \omega)$, when flexible feedbacks are introduced.

Effect of flexible feedbacks by the first and third derivatives so as the effect of the feedbacks by the second and fourth derivatives are mutually opposite. Feedbacks by the third and fourth derivatives have a little effect on the initial (low frequency) part of the characteristic and significantly distort its high-frequency part.

In the RCAV control systems the forced oscillations have a low frequency spectrum, depending on the perturbation and control signals. Therefore, for correction the quality generally are used the feedbacks by the first two derivatives - by speed and by acceleration of the control object.

Application of the feedback by the first derivative (by the velocity) increases the stability margins in modulus $h_{E a^{\prime}}>h_{E a}$ and phase $\gamma_{a^{\prime}}>\gamma_{a}$, shifting up (Fig, 2) the characteristic $E_{\mathrm{ol}}(j \omega)$. It is necessary to mean that in this case the tracking error is increases.

Application of the feedback by the second derivative (by the acceleration) increases the stability margin in modulus, but reduces the stability margin in phase, since the characteristic 
$E_{\text {ol }}(j \omega)$ is shifting to the left due to the component $-k_{2} \omega^{2}$. In this case the stability region is expanding, but at the same time increases the transient recovery time. This demonstrate, in particular, a decreasing of the frequency $\omega_{\mathrm{p}}$ of the positiveness of characteristic $P(\omega)$, with which the transient recovery time related by the ratio $t_{\mathrm{tr}}>\pi / \omega_{\mathrm{p}}$.

What was said above should be considered when selecting the type of flexible correcting feedback in stabilization systems.

Serial corrective devices are usually realized in the form of differentiating or integro-differentiating passive DC circuits. The differentiating circuits along with the expansion of the area of stability of the system improve its action in time, providing the forcing of transients.

Figure 3 shows the effect from the sequential correction circuit (SCC) on the region of stability of the control system. SCC moves to the right the low-frequency stability border, thus enabling to regulate the system on a larger stiffness. In the same figure is compared the ideal and the real differentiating circuits. Comparison demonstrates that the non-ideality of the differentiating circuit reduces its impact on the effectiveness of the system.

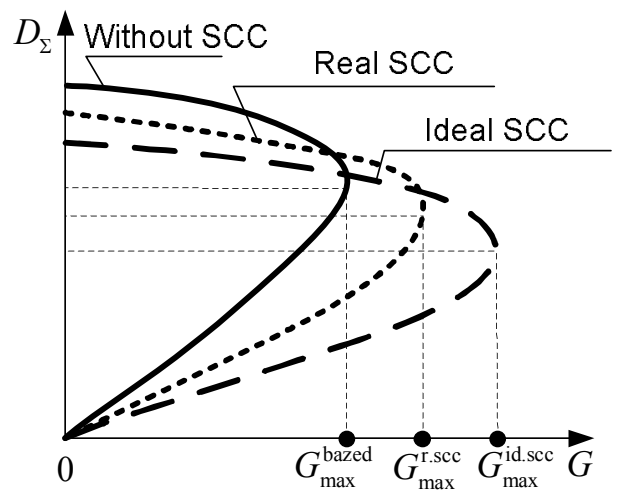

Fig. 3. Impact of SCC on the stability region.

Raising the margin of stability of the system while simultaneously forcing of the transients - important advantage of the successive differentiating contours. The widespread use of these contours for the correction the quality of system is also caused by the simplicity of their constructive realization.

The main disadvantages of the successive differentiating contours include:

- significant (by 10 times or more) reduction of the amplification coefficient of control system, requiring a corresponding increase in the transfer coefficients of other units or the introduction of special amplifiers;

- high sensitivity to interference (noises);
- the difficulty of implementing of contours for AC control signals and therefore the necessity to have in the system the phase-sensitive amplifiers .

Much less often differentiating circuits apply the integrating SCC. Application of the integrating circuit (ideal) allows to increase an order of magnitude system astatism, but leads to deterioration of its stability.

In order to choose one or the other type of corrective device and determine its parameters, you must compare the original (uncorrected) system with the desired system. Comparisons are usually conducted on logarithmic amplitude and phase frequency characteristics.

When correction is serial transfer function of the desired (corrected) open-loop system will be equal to:

$$
W_{\text {ol.des }}(s)=W_{\text {ol }}(s) W_{\mathrm{c}}(s) \text {, }
$$

where $W_{\mathrm{ol}}(s)$ is the transfer function of the original (uncorrected) system; $W_{\mathrm{c}}(s)$ is the transfer function of SCC.

Hence the amplitude phase frequency characteristic of the correcting device can be determined from the from the relation:

$$
W_{\mathrm{c}}(j \omega)=\frac{W_{\mathrm{olddes}}(j \omega)}{W_{\mathrm{ol}}(j \omega)} .
$$

Proceeding to logarithmic and phase frequency characteristics, we have:

$$
\left.\begin{array}{l}
L_{\mathrm{c}}(\omega)=L_{\text {oldes }}(\omega)-L_{\mathrm{ol}}(\omega) ; \\
\varphi_{\mathrm{c}}(\omega)=\varphi_{\text {ol.des }}(\omega)-\varphi_{\mathrm{ol}}(\omega) .
\end{array}\right\}
$$

Thus, the logarithmic and phase frequency characteristics of the serial correcting device determines as the difference between the desired and original characteristics. Methods for determining the desired frequency characteristics of control systems are set forth in [1].

Typically, determination of $L_{\mathrm{c}}(\omega)$ is made graphically (Fig. 4).

In case of parallel correction, transfer function of desired (corrected) open-loop system will be

$$
W_{\text {oldes }}(s)=\frac{W_{\text {ol }}(s)}{1+W_{\text {cov }}(s) W_{\mathrm{fbc}}(s)},
$$

where $W_{\text {cov }}(s)$ is the transfer function of the elements, that 
are covered by the feedback correction; $W_{\mathrm{fbc}}(s)$ is the transfer function of the feedback correction.

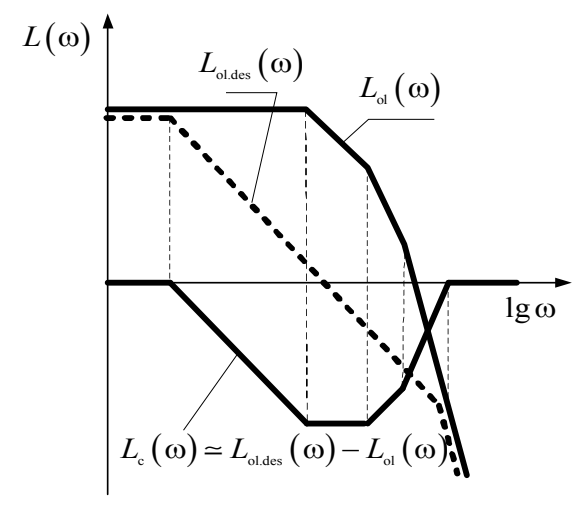

Fig. 4. LAFC determination of the correcting device.

Taking into account that by any method of correction must be obtained the same desirable characteristic, will equate (2) and (4), substituting $s=j \omega$ :

$$
W_{\mathrm{c}}(j \omega)=\frac{1}{1+W_{\mathrm{cov}}(j \omega) W_{\mathrm{fbc}}(j \omega)}
$$

For the frequency range in which

$$
W_{\text {cov }}(j \omega) W_{\mathrm{fbc}}(j \omega) \ll 1
$$

correction is not required, since $W_{\mathrm{c}}(j \omega) \cong 1$.

For the frequency range when

$$
W_{\mathrm{cov}}(j \omega) W_{\mathrm{fbc}}(j \omega) \gg 1
$$

will have

$$
L_{\mathrm{c}}(\omega)=-\left[L_{\mathrm{cov}}(\omega)+L_{\mathrm{fbc}}(\omega)\right]
$$

Having defined by equation (3) the characteristic $L_{\mathrm{c}}(\omega)$ and knowing the characteristic $L_{\text {cov }}(\omega)$, we can find:

$$
L_{\mathrm{fbc}}(\omega)=-\left[L_{\mathrm{cov}}(\omega)+L_{\mathrm{c}}(\omega)\right]
$$

Using special tables, by known characteristics $L_{\mathrm{c}}(\omega)$ or $L_{\mathrm{fbc}}(\omega)$, it is possible to select the scheme and find the parameters of the serial or parallel correcting device.

\section{CONCLUSIONS}

The main difficulty to optimize the quality of functioning of the RCAV is the synthesis of corrective devices of their control systems.

The present analysis of the properties of serial and parallel corrective devices and algorithms for calculating their frequency characteristics can be useful for novice developers RCAV control systems.

Information about calculating the desired frequency characteristics of the control systems by the type of the transient process or by the minimum control error may be found in authors works for example in [1].

\section{REFERENCES}

[1] A.K. Ablesimov, L.V. Pogribnyak, M.A. Pylypenko, and N.V. Usenko, "Methods of Determining the Desired Frequency Characteristics of the Automatic Control Systems." 2016 IEEE 4th International Conference "Methods and Systems of Navigation and Motion Control"“. October 1820, 2016, Kyiv, Ukraine.

[2] A. K. Ablesimov, 2014. Course of the theory of automatic control. Kyiv: Osvita Ukrainy. 270 p. (in Ukrainian). 